\title{
Analysis of passive tibio-femoral joint movement of Beagle dogs during flexion in cadaveric hind limbs without muscle
}

J. Vet. Med. Sci.

82(2): 148-152, 2020

doi: 10.1292/jvms.18-0501

Received: 20 August 2018

Accepted: 2 December 2019 Advanced Epub:

13 December 2019

\author{
Tom ICHINOHE ${ }^{1) *}$, Nobuo KANNO ${ }^{2)}$, Yasuji HARADA ${ }^{2)}$, Yukihiro FUJTAA ${ }^{1)}$, \\ Hiromichi FUJIE') and Yasushi HARA ${ }^{2)}$ \\ 1)The Laboratory of Small Animal Surgery, Azabu University, 1-17-71 Fuchinohe, Chuo, Sagamihara-shi, \\ Kanagawa 252-0206, Japan \\ 2)The Laboratory of Veterinary Surgery, Nippon Veterinary and Life Science University, 1-7-1 Kyonan-cho, \\ Musashino-shi, Tokyo 180-8602, Japan \\ 3)Biomechanics Laboratory, Faculty of System Design, Tokyo Metropolitan University, 6-6 Asahigaoka, \\ Hino-shi, Tokyo 191-0065, Japan
}

\begin{abstract}
The aim of this study was to evaluate the normal range of motion of the canine tibiofemoral joint and the movement of the tibia relative to the femur, especially the internal/ external rotation under flexion. Right stifle joints were harvested from eight skeletally mature Beagle dogs, which were euthanized for reasons unrelated to this study. All muscle tissue was removed from the limbs prior to testing. Flexion and extension tests were conducted using a robotic manipulator with six degrees-of-freedom. Cranial/caudal and medial/lateral displacement and varus/valgus and internal/external rotation were measured at various degrees of flexion. We observed that the tibia rotated internally at an increasing flexion angle with mean peak internal and external rotations of $20.0 \pm 13.8^{\circ}$ and $4.5 \pm 3.6^{\circ}$, respectively. The tibia also tended to displace cranially at an increasing flexion angle, with a mean peak cranial displacement of $8.9 \pm 4.4 \mathrm{~mm}$; there was minimal medial displacement when increasing the flexion angle. Valgus rotation also tended to occur at an increasing flexion angle. During the flexion of the canine stifle joint, approximately $20^{\circ}$ of internal tibial rotation occurred around the longitudinal axis, along with a rollback motion involving the cranial displacement of the tibia.
\end{abstract}

KEY WORDS: canine stifle joint, flexion/extension, internal/external rotation of the tibia

Patellar luxation (PL) is reported as a postoperative complication of tibial plateau leveling osteotomy (TPLO), which is one of the standard surgeries for cranial cruciate ligament rupture (CCLR) [2]. A proposed cause of PL is the rotational instability of the tibiofemoral joint due to the absence of an intact cranial cruciate ligament (CCL) [2]. However, verification of this cause is difficult because the range of rotation in a normal canine tibiofemoral joint remains unclear.

The tibiofemoral joint is a complex hinge joint with primary motion in two planes. Rotational movements of the tibia on the femur occur around the longitudinal axis, while flexion and extension occur on the transverse axis [11]. In humans, many studies have reported that internal rotation of the tibia is coupled with flexion of the tibiofemoral joint $[12,14,18-20]$. Internal rotation of the human tibia during flexion strains the vastus lateralis (the main extensor in the quadriceps) and increases the efficiency of its traction $[8,10]$. Possible causes of internal rotation of the tibia during flexion of the tibiofemoral joint are as follows: 1) a change in tension of the lateral collateral ligament (LCL) with flexion and extension of the tibiofemoral joint [18]; 2) a difference in conformation between the lateral and medial compartments of the tibiofemoral joint, associated with the joint surface morphology $[8,13]$; and 3) a difference in motion of the lateral and medial femoral condyles on the tibia under flexion due to the influence of the internal rotators (e.g., the sartorius, semitendinosus, semimembranosus, gracilis, and popliteus muscles) [8]. The observation of internal rotation during flexion in humans supports the argument that the tibia follows a rotational path in passive flexion; however, no study to date has described the path completely in dogs. The aims of this study were to evaluate the normal range of motion of the canine tibiofemoral joint and to evaluate the movement of the tibia relative to the femur, especially internal/external rotation, during flexion of the canine stifle joint. 


\section{MATERIALS AND METHODS}

\section{Animals}

Cadaveric stifle joints ( $\mathrm{n}=8$, right hind limbs) were harvested from Beagle dogs who had attained normal skeletal maturity (body weight: $10.1 \pm 1.0 \mathrm{~kg}$; age: $3.3 \pm 4.5$ years; five males, three females) and had been euthanized for reasons unrelated to this study. Euthanasia of the dogs was performed in accordance with the Guidelines for Care and Use of Laboratory Animals, Nippon Veterinary and Life Science University (approval No. 09-58 and 09-78). Normal clinical and anatomical status of the stifle joints, with absence of signs of degenerative joint disease, was confirmed through radiographic and orthopedic screening. To measure the anatomic lateral distal femoral angle (aLDFA, Fig. 1A) $[4,16]$ and the mechanical medial proximal tibial angle (mMPTA, Fig. 1B) $[3,4]$, craniocaudal radiographs were acquired from all specimens. This showed the appearance of the walls of the intercondylar notch and the slight protrusion of the corticocancellous tip of the lesser trochanter [4]. To measure the tibial plateau angle (TPA, Fig. 1C), mediolateral radiographs were acquired from all specimens with a 100-cm distance between the stifle and the X-ray tube and with the stifle joints positioned at $90^{\circ}$ of flexion [15]. All radiographs showed superimposition of the femoral condyles [15]. The aLDFA, mMPTA, and TPA were measured as previously described $[3,4,15,16]$. The average aLDFA was $94.5 \pm 2.6^{\circ}$. The average TPA and mMPTA were $29.3 \pm 3.5^{\circ}$ and $92.1 \pm 3.2^{\circ}$, respectively.

\section{Experimental procedures}

The femur of each specimen was transected at the coxofemoral joint, and the tibia and fibula at the tarsocrural joint. All soft tissues, other than the stifle joint capsule and ligaments supporting the stifle joint, were removed. The specimens were then wrapped in gauze soaked in lactated Ringer's solution and stored at $-80^{\circ} \mathrm{C}$ until preparation. The specimens were thawed at room temperature overnight prior to preparation. The femur and the tibia were embedded in acrylic resin (OSTRON2; GC Corp., Tokyo, Japan) to be positioned in the custom-made dog stifle clamps of the testing system [9]. The specimens were sprayed with lactated Ringer's solution to prevent drying throughout the experimental procedure. The simulator used in this study comprised a manipulator with six degrees-of-freedom (6-DOF) with a 6-DOF universal force/moment sensor (UFS) and a simulator based on the joint coordinate system reported by Grood and Suntay [7]. The stifle coordinate system was used to define the femur and tibia in each specimen. The 6-DOF motions in the stifle joint are as follows: flexion-extension (FE), medial-lateral (ML) translation, varus-valgus (VV) rotation, cranial-caudal $(\mathrm{CrCa})$ translation, internal-external (IE) rotation, and proximal-distal (PD) translation [5]. The manipulator with 6-DOF included 2 different mechanisms: the upper mechanism (movement at two translational and three rotational axes); and the lower mechanism (movement at one translational axis). The tibial clamp was fixed to the upper mechanism via the UFS, and the femoral clamp was fixed to the lower mechanism. This system can be used to calculate the displacement and

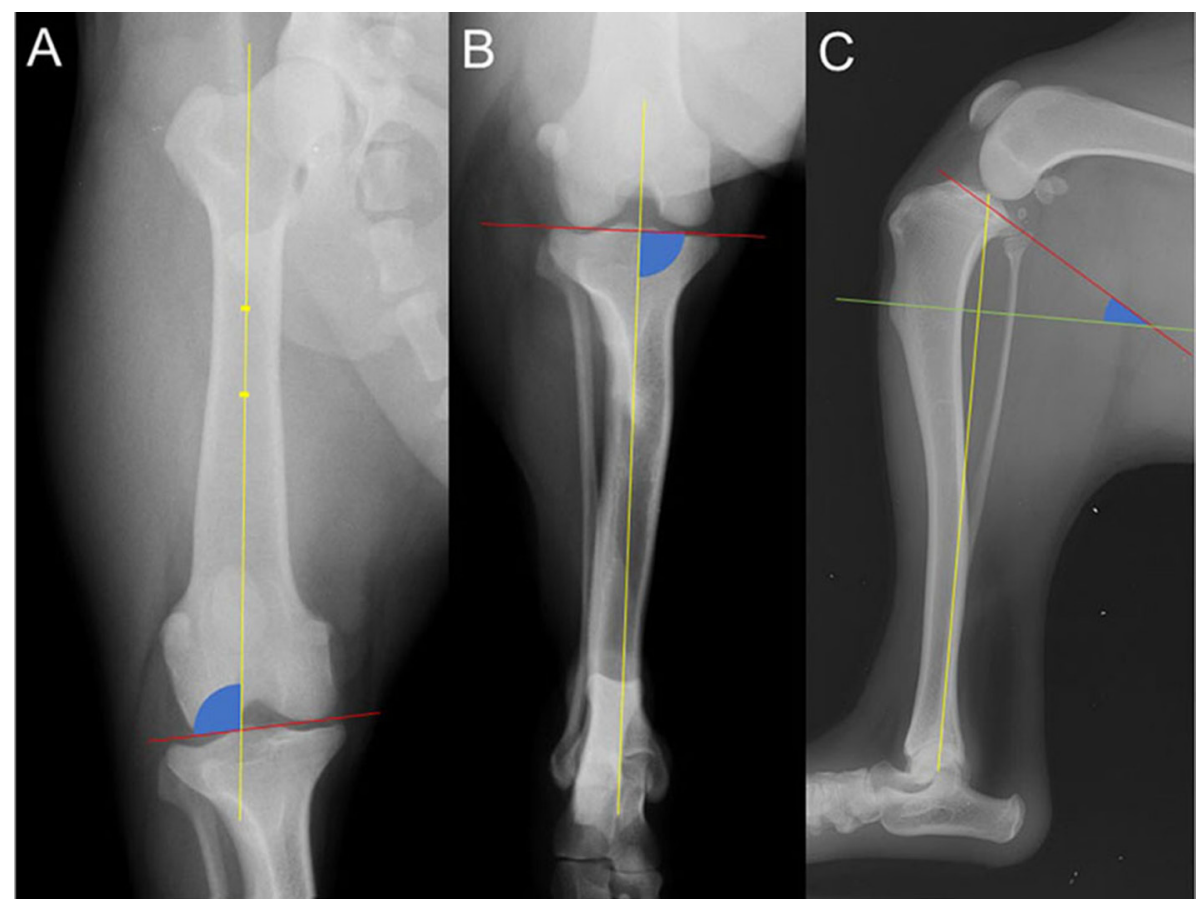

Fig. 1. The measurement on the radiography. (A) The measurement scheme of the anatomic lateral distal femoral angle (aLDFA). The aLDFA (blue angle) is the intersecting angle of anatomic axis and distal joint orientation line of the femoral joint. (B) The measurement scheme of the mechanical medial proximal tibial angle (mMPTA). The angle between the mechanical axis and joint orientation line is measured on the proximomedial aspect of the tibia to determine the mMPTA (blue angle). (C) The determination of the tibial plateau angle (TPA). The angle between the tibial plateau line and a line perpendicular to the tibial axis (green line) is measured to determine the TPA (blue angle). 
force/moment applied to the tibiofemoral joint, and for the coordinate transformation of data obtained through the UFS [5]. Further, it can precisely simulate complex loading scenarios in the stifle (e.g., anterior force combined with joint compressive force loading at relatively low speed) while allowing natural joint motion [6].

The system was initially set up so all UFS outputs were zero. The stifle was extended until it reached 0.5 -Nm of extension during FE in the force control (i.e., overextension). The joint angle under this condition was measured and the tibial coordinate system was defined [9]. In each specimen, the flexion angle was determined by subtracting the angle formed by the femur and the tibia from $180^{\circ}$. The average flexion angle was $25.2 \pm 1.7^{\circ}$, following overextension of the stifle. To prevent the tissue creep effect, the stifle was extended and flexed three times from overextension to $125^{\circ}$ of flexion. ML translation, $\mathrm{VV}$ rotation, $\mathrm{CrCa}$ translation, IE rotation, and PD translation were determined with no applied force (to allow natural movement). Flexion of the knee was conducted between overextension and $125^{\circ}$ of flexion in the displacement control. CrCa displacement and IE rotation were measured at $30^{\circ}, 45^{\circ}, 60^{\circ}, 75^{\circ}, 90^{\circ}, 105^{\circ}$, and $120^{\circ}$ of flexion. In addition, ML displacement and VV rotation were measured.

\section{Statistical analysis}

Results are reported as the mean \pm standard deviation (SD). Statistical comparisons between degree of displacement at each angle were performed using one-way analysis of variance (ANOVA) for repeated measures (BellCurve for Excel; Social Survey Research Information Co., Ltd., Tokyo, Japan). For all tests, values of $P<0.05$ were considered significant.

\section{RESULTS}

\section{Tibial IE rotation}

The tibia tended to rotate first externally, then internally with increasing flexion angle. The mean peak internal rotation was $20.0 \pm 13.8^{\circ}$ (range: $0.2-36.1^{\circ}$ ), and the mean peak external rotation was $4.5 \pm 3.6^{\circ}\left(0.4-10.4^{\circ}\right)$. There were significant differences between the degree of internal rotation at each angle ( $P=0.003$, Fig. 2).

\section{Tibial $\mathrm{CrCa}$ displacement}

Cranial tibial displacement tended to occur with increasing flexion angle. The mean peak cranial displacement was $8.9 \pm 4.4 \mathrm{~mm}$ (2.4-16.2 mm). There were significant differences between the distance of cranial displacement at each angle $(P<0.001$, Fig. 3).

\section{Tibial ML displacement and VV rotation}

There was minimal medial tibial displacement with increasing flexion angle. The mean peak medial displacement was $1.3 \pm$ $0.7 \mathrm{~mm}(0.4-2.6 \mathrm{~mm})$ and the mean peak lateral displacement was $0.2 \pm 0.3^{\circ}\left(-0.1-0.8^{\circ}\right)$. There were no significant differences between the amount of medial displacement observed at each angle $(P=0.203$, Fig. 4$)$. Moreover, the tibias tended to show varus rotation first, followed by valgus rotation with increasing flexion angle. The mean peak varus rotation was $3.8 \pm 4.0^{\circ}\left(0.0^{-10.1^{\circ}}\right)$ and the mean peak valgus rotation was $7.0 \pm 6.5^{\circ}\left(0.6-19.0^{\circ}\right)$. There were significant differences between the degree of varus rotation at each angle $(P=0.003$, Fig. 5).

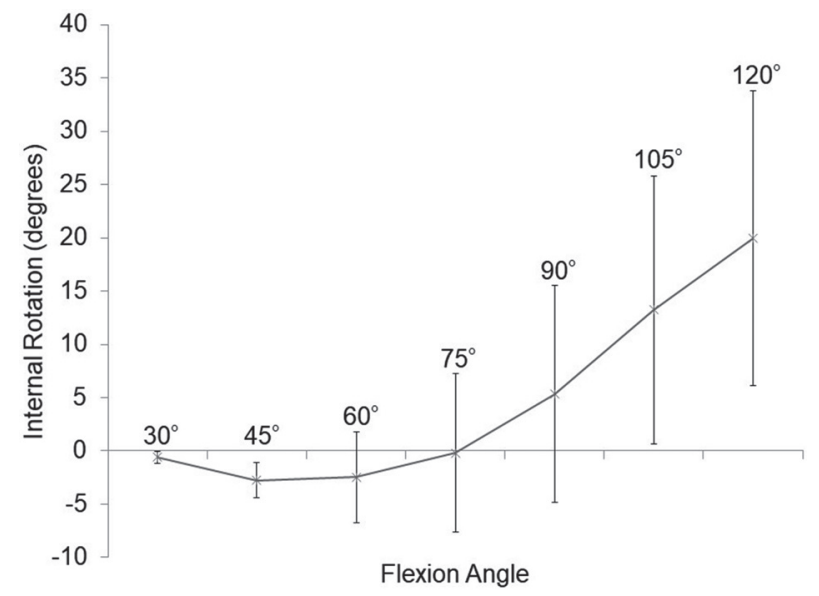

Fig. 2. Tibial internal rotation around longitudinal axis under flexion. The tibia tended to rotate first externally, then internally with increasing flexion angle. Significant differences were detected about degree of internal rotation $(P=0.003)$.

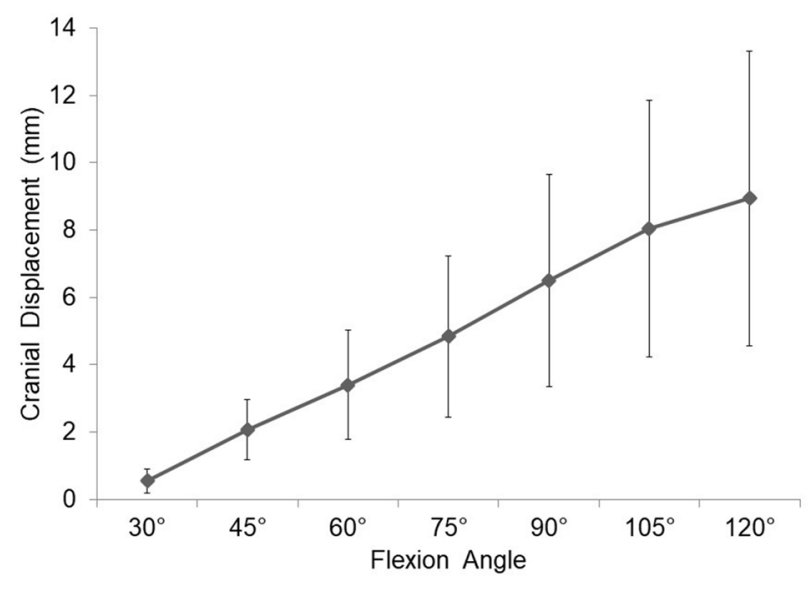

Fig. 3. Tibial cranial displacement with flexion. Cranial tibial displacement tended to occur with increasing flexion angle. Significant differences were detected about the amount of cranial displacement $(P<0.001)$. 


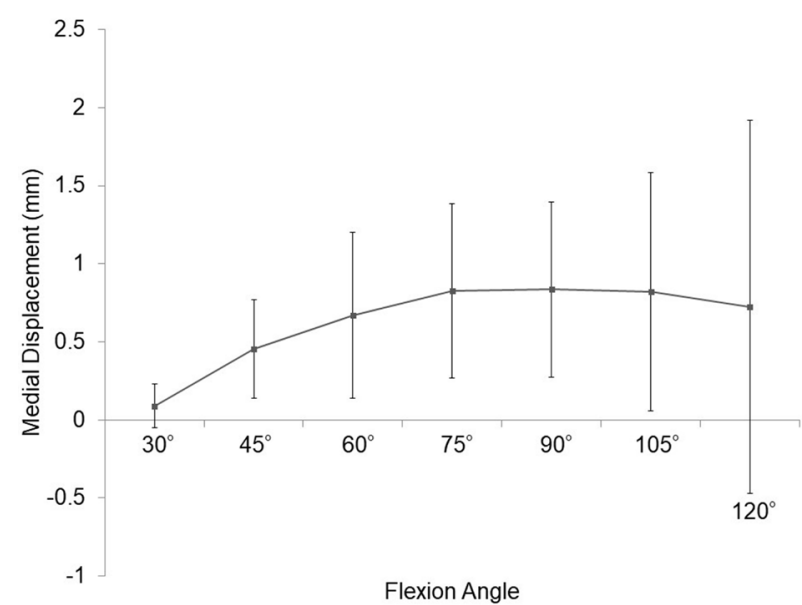

Fig. 4. Tibial medial displacement under flexion. There was minimal medial tibial displacement with increasing flexion angle. There were no significant differences between the amount of medial displacement at any of the angles $(P=0.203)$.

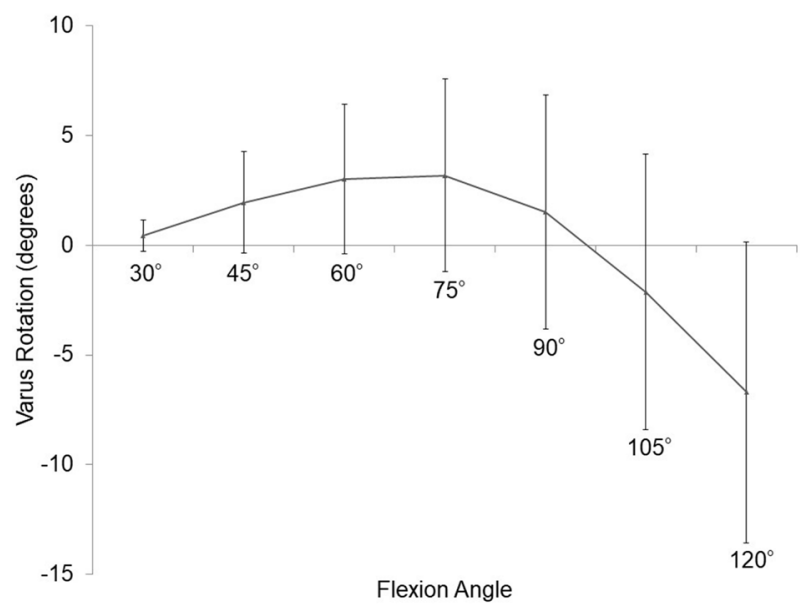

Fig. 5. Tibial varus rotation under flexion. The tibia tended to show varus rotation first, followed by valgus rotation, with increasing flexion angle. Significant differences were detected about the amount of varus movement $(P=0.003)$.

\section{DISCUSSION}

It was confirmed that an increase of internal tibial rotation occurs with increasing flexion angle in the canine stifle joint, as seen in humans. In addition, we observed that the tibia rotated internally relative to the femur, by approximately $20^{\circ}$, with flexion of the stifle joint. In humans, internal rotation is reported to range from 8.5 to $37^{\circ}[12,17]$. It was thought that a similar range of motion would be observed in the canine stifle joint; however, this was not observed. The large variation reported in humans may be attributable to differences in test apparatuses, conventions for the representation of knee rotation, and definitions of coordinate systems.

The results of the current study also show that the canine tibia is displaced cranially under stifle flexion. The presence of femoral roll-back is likely, whereby the femur translates caudally, relative to the tibia as the knee flexes [8, 19]. It has been reported that this may be beneficial in maximizing flexion range by delaying contact between the caudal proximal tibia and corresponding caudal distal femur. It also enlarges the lever arm of the stifle extensor mechanism, thus increasing the mechanical advantage during stifle extension [19]. It is suggested that the same phenomenon occurs in dogs as in humans.

Vasseur et al. [18] investigated the change in LCL tension of the canine tibiofemoral joint under flexion and extension. The LCL relaxes under flexion of the joint due to the shortening of the distance between the area proximal to the insertion site of the popliteus tendon and the head of the fibula, which are the insertion sites of the LCL. This allows caudal displacement of the lateral condyle. Conversely, the medial collateral ligament (MCL) remains tense under both extension and flexion because the distance between cranial insertion sites does not change. This regulates the caudal displacement of the medial condyle, compared with that of the lateral condyle. Thus, the tibia rotates internally around the longitudinal axis [18]. Moreover, since the articular surface of the lateral condyle is shorter than that of the medial condyle, the lateral condyle exhibits a greater degree of caudal motion than does the medial condyle. Additionally, the slide accompanying rolling is larger for the medial condyle than that for the lateral condyle as the medial condyle reaches the caudal end of the tibia, stops, and starts sliding earlier than does the lateral condyle [13]. This suggests a similarity in the mechanism of internal tibial rotation between the canine stifle and the human knee joint. However, morphological differences between the lateral and medial femoral condyles were not assessed in this study. More morphological evaluation of the canine femoral condyle is required to characterize this further.

Differences in the conformation between the medial and lateral compartments of the tibiofemoral joint have not been assessed in this study. However, the femur is known to roll-back under flexion; this was observed in this study. Differences in the length of the medial and lateral condyles in humans are thought to be responsible for the differences in their respective movements within the tibiofemoral joint $[8,13]$. The displacement of the meniscus on the tibia is likely to affect tibial internal rotation; however, this was not verified in this study. While the MCL forms strong attachments to the joint capsule and medial meniscus, attachments have not been observed between the LCL and lateral meniscus [18]. This is thought to allow greater posterior translation of the lateral meniscus, compared to the medial [21]. In addition, the meniscofemoral ligament of the lateral meniscus binds the medial side of the medial femoral condyle and the lateral meniscus [11]. Consequently, although both medial and lateral menisci show caudal translation along with the femoral condyles during flexion, less translation of the medial meniscus occurs compared with the lateral. This is expected to regulate the caudal displacement of the medial condyle, causing the tibia to rotate internally, with the center of rotation at the medial meniscus.

A limitation of this study is that the robotic system used was designed to study the biomechanics of the human knee. The system generates a reproducible motion on a loaded limb in a quasi-static manner. The range of internal rotation under flexion of the 
tibiofemoral joint in Beagle dogs was determined. Due to possible breed-related differences, and differences between the right and left stifle joints, it may be necessary to evaluate the range of motion in other breeds and in left stifle joints. Another limitation was that the standard deviation of the data, especially during flexion, might be too large. This may be due to the luxation of the CCL, LCL, and soft tissues surrounding the stifle, (e.g., the joint capsule during flexion) [1, 18]. The contraction of the rotator muscles of the stifle could affect internal tibial rotation. The stifle flexor muscles are classed as internal rotators and external rotators; therefore, these muscles produce rotational movement of the tibia during knee flexion [8]. However, the robotic system used in our study was developed for static analysis, not considering dynamic changes in the stifle that may occur during normal motion.

In conclusion, our results indicate that internal tibial rotation around the longitudinal axis, of approximately $20^{\circ}$, occurs under flexion in the canine stifle joint. The results of this study may be useful in investigating the causes of diseases that accompany rotatory instability, such as PL, and in developing prevention strategies.

\section{REFERENCES}

1. Arnoczky, S. P. and Marshall, J. L. 1977. The cruciate ligaments of the canine stifle: an anatomical and functional analysis. Am. J. Vet. Res. 38: 1807-1814. [Medline]

2. Arthurs, G. I. and Langley-Hobbs, S. J. 2007. Patellar luxation as a complication of surgical intervention for the management of cranial cruciate ligament rupture in dogs. A retrospective study of 32 cases. Vet. Comp. Orthop. Traumatol. 20: 204-210. [Medline] [CrossRef]

3. Dismukes, D. I., Tomlinson, J. L., Fox, D. B., Cook, J. L. and Song, K. J. 2007. Radiographic measurement of the proximal and distal mechanical joint angles in the canine tibia. Vet. Surg. 36: 699-704. [Medline] [CrossRef]

4. Fox, J. D. and Tomlinson, J. L. 2011. Principles of angular limb deformity correction. pp. 657-668. In: Veterinary Surgery: Small Animal, 1st ed. Vol. 1 (Tobias, K. M. and Johnston, S. A. eds.), Saunders, Philadelphia.

5. Fujie, H., Livesay, G. A., Fujita, M. and Woo, S. L. 1996. Forces and moments in six-DOF at the human knee joint: mathematical description for control. J. Biomech. 29: 1577-1585. [Medline] [CrossRef]

6. Fujie, H., Sekito, T. and Orita, A. 2004. A novel robotic system for joint biomechanical tests: application to the human knee joint. J. Biomech. Eng. 126: 54-61. [Medline] [CrossRef]

7. Grood, E. S. and Suntay, W. J. 1983. A joint coordinate system for the clinical description of three-dimensional motions: application to the knee. J. Biomech. Eng. 105: 136-144. [Medline] [CrossRef]

8. Kapandji, A. I. 2010. The knee. pp. 66-155. In: Physiology of the Joints, 6th ed., Vol. 2, Lower Limb., Elsevier, Amsterdam.

9. Kanno, N., Hara, Y., Fukano, S., Fujie, H., Ochi, H., Fujita, Y., Yasuji, H., Nezu, Y., Yogo, T. and Tagawa, M. 2014. Tibial displacement with stifle joint flexion and cranial cruciate ligament transection in the dog. An ex vivo study using a robotic simulator. Vet. Comp. Orthop. Traumatol. 27: 277-284. [Medline] [CrossRef]

10. Lieb, F. J. and Perry, J. 1968. Quadriceps function. An anatomical and mechanical study using amputated limbs. J. Bone Joint Surg. Am. 50: 1535-1548. [Medline] [CrossRef]

11. Liebich, H. G., Koing, H. E. and Maierl, J. 2007. Hindlimb or pelvic limb. pp. 223-288. In: Veterinary Anatomy of Domestic Mammals 4th ed., Schattauer, Stuttgart.

12. Markolf, K. L., Mensch, J. S. and Amstutz, H. C. 1976. Stiffness and laxity of the knee--the contributions of the supporting structures. A quantitative in vitro study. J. Bone Joint Surg. Am. 58: 583-594. [Medline] [CrossRef]

13. Nakamura, R. and Saito, H. 1983. Stifle joint motion. pp. 193-206. In: Fundamental Kinesiology, 2nd ed., Ishiyaku Publishers Inc., Tokyo.

14. Shoemaker, S. C., Adams, D., Daniel, D. M. and Woo, S. L. 1993. Quadriceps/anterior cruciate graft interaction. An in vitro study of joint kinematics and anterior cruciate ligament graft tension. Clin. Orthop. Relat. Res. 379-390. [Medline] [CrossRef]

15. Slocum, B. and Slocum, T. D. 1993. Tibial plateau leveling osteotomy for repair of cranial cruciate ligament rupture in the canine. Vet. Clin. North Am. Small Anim. Pract. 23: 777-795. [Medline] [CrossRef]

16. Tomlinson, J., Fox, D., Cook, J. L. and Keller, G. G. 2007. Measurement of femoral angles in four dog breeds. Vet. Surg. 36: 593-598. [Medline] [CrossRef]

17. Trent, P. S., Walker, P. S. and Wolf, B. 1976. Ligament length patterns, strength, and rotational axes of the knee joint. Clin. Orthop. Relat. Res. 263-270. [Medline]

18. Vasseur, P. B. and Arnoczky, S. P. 1981. Collateral ligaments of the canine stifle joint: anatomic and functional analysis. Am. J. Vet. Res. 42: 1133-1137. [Medline]

19. Williams, A. and Logan, M. 2004. Understanding tibio-femoral motion. Knee 11: 81-88. [Medline] [CrossRef]

20. Wilson, D. R., Feikes, J. D., Zavatsky, A. B. and O’Connor, J. J. 2000. The components of passive knee movement are coupled to flexion angle. J. Biomech. 33: 465-473. [Medline] [CrossRef]

21. Yao, J., Lancianese, S. L., Hovinga, K. R., Lee, J. and Lerner, A. L. 2008. Magnetic resonance image analysis of meniscal translation and tibiomenisco-femoral contact in deep knee flexion. J. Orthop. Res. 26: 673-684. [Medline] [CrossRef] 